\title{
Proposal of Thickness Inspection Method of Opposite-side Nickel Layer on Nickel-coated Steel Plate Using Pulsed Magnetic Field
}

\author{
Yuji Gotoh $^{1 *}$ and Makoto Tohara ${ }^{2}$ \\ ${ }^{1}$ Department of Innovative Engineering, Faculty of Science and Technology, Oita University, \\ 700 Dannoharu, Oita 870-1192, Japan \\ ${ }^{2}$ Department of Materials Science and Production Engineering, Oita University, \\ 700 Dannoharu, Oita 870-1192, Japan
}

(Received April 22, 2019; accepted July 1, 2019)

Keywords: nickel layer, nickel steel plate, opposite side, pulsed magnetic field, nondestructive inspection

Since nickel-coated steel is widely used to prevent rust, it is used in large rolled steel plates in paper mills. It is necessary to inspect the thickness of the nickel layer on nickel-coated steel in the production process to guarantee quality. Moreover, in a real plant, the inspection of the nickel layer on the opposite side of a rolled steel plate is required. The conductivity of the nickel layer in the nickel-coated steel plate is higher than that of the mother steel, and its permeability is lower than that of the mother steel. Therefore, the thickness of the nickel layer can be estimated on the basis of the differences in these electromagnetic properties. In this paper, an electromagnetic inspection method for measuring the thickness of the nickel layer on the opposite side using a pulsed magnetic field is proposed. The detailed phenomenon of the inspection method is examined by the 3D nonlinear finite element method (FEM) considering the hysteresis magnetization curves of both nickel and the mother steel plate. It is shown that the electromagnetic inspection of the thickness of the nickel layer is possible by using the output voltage of a search coil of the proposed sensor.

\section{Introduction}

There is a rolling process for paper in a paper mill. In the rolling process, nickel-coated steel is used in a large rolled steel plate. It is necessary to inspect the thickness of the nickel layer during the production process to guarantee quality. ${ }^{(1,4)}$ In a real manufacture plant, the thickness of the nickel layer on the opposite side of a rolled steel plate should be inspected. An $\mathrm{X}$-ray measurement method ${ }^{(5)}$ is generally used for thickness inspection on that side. However, X-rays do not permeate up to the nickel layer on the opposite side of a thick rolled steel plate.

The conductivity of a nickel layer in nickel-coated steel is higher than that of the mother steel (SS400 steel), and its permeability is lower than that of the mother steel. Therefore, the thickness of the nickel layer can be estimated on the basis of the differences in these electromagnetic properties.

*Corresponding author: e-mail: goto-yuuji@oita-u.ac.jp https://doi.org/10.18494/SAM.2019.2411 
In this paper, an electromagnetic inspection method for measuring the thickness of the nickel layer on the opposite side of a nickel-coated steel plate using a pulsed magnetic field is proposed. The detailed phenomenon of the inspection method is examined by the 3D nonlinear finite element method (FEM) ${ }^{(6)}$ considering the hysteresis magnetization curves of both nickel and the mother steel (SS400 steel) in the nickel-coated steel plate. In addition, the method is experimentally verified.

\section{Electromagnetic Properties of Nickel and Mother Steel}

The hysteresis magnetization curves of the nickel and mother steel (SS400 steel) are measured using an electromagnet as shown in Fig. 1. A column specimen is placed between pole pieces and magnetized by a magnetic field of $0.1 \mathrm{~Hz}$. The flux density in the specimen is measured using a search coil that is connected to a flux meter, and the flux density $B$ inside the specimen is measured. The magnetic field strength $H$ is measured using a transverse-type Hall element. ${ }^{(7)}$ Figure 2 shows the hysteresis magnetization curves of the nickel and mother steel (SS400 steel) with a magnetic field range of $\pm 3000 \mathrm{~A} / \mathrm{m}$. The figure denotes that the permeability of nickel is lower than that of the SS400 mother steel.

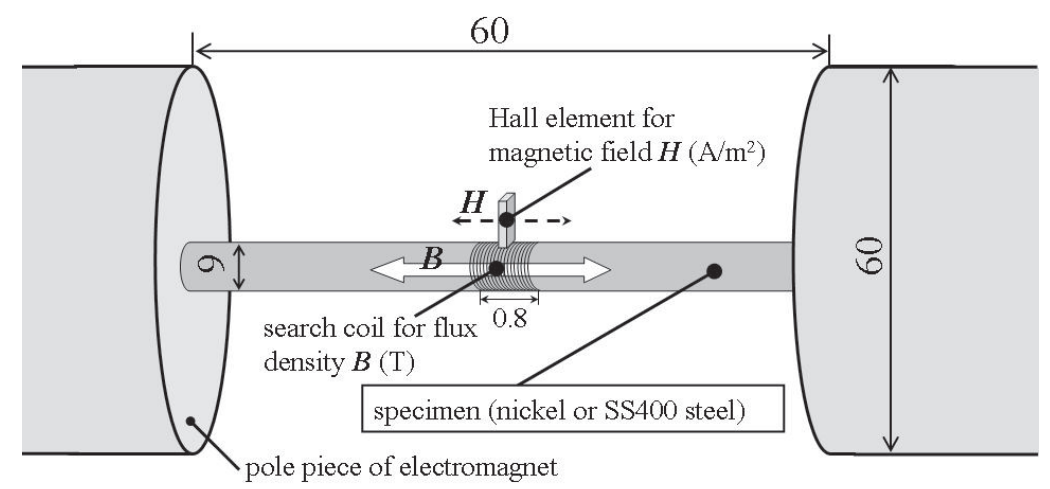

Fig. 1. Electromagnet for measuring hysteresis magnetization curves of nickel and mother steel (SS400 steel) (excitation frequency, $0.1 \mathrm{~Hz}$; unit, $\mathrm{mm}$ ).

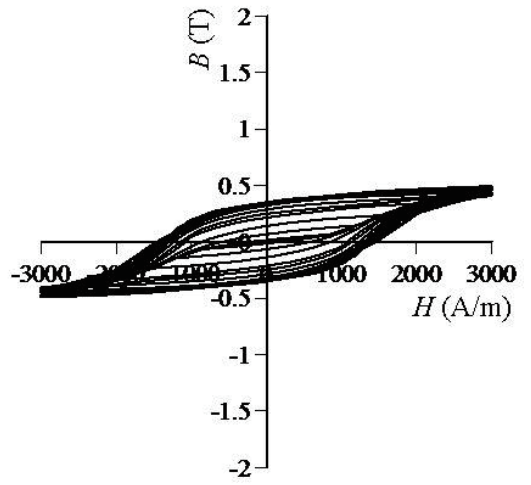

(a)

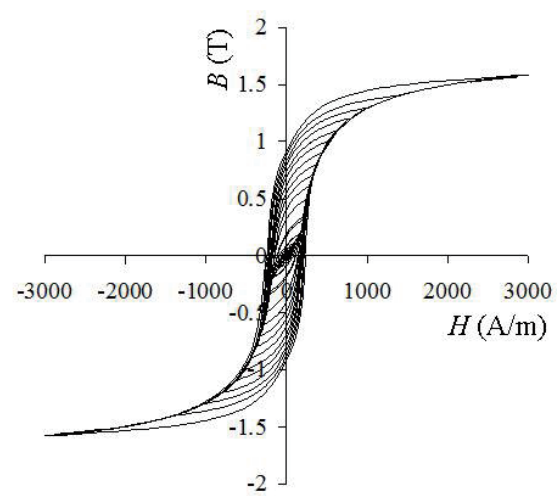

(b)

Fig. 2. Hysteresis magnetization curves of (a) nickel and (b) mother (SS400) steel (range of magnetic field $H$ : $\pm 3000 \mathrm{~A} / \mathrm{m})$. 
The conductivities of nickel and SS400 steel specimens are measured using the Kelvin double-bridge circuit (Table 1). The conductivity of nickel is 1.6 times that of SS400 steel.

\section{Inspection of Thickness of Nickel Layer by Pulsed Magnetic Field}

\subsection{Inspection model}

Figure 3 shows the 1/2 domain of the proposed electromagnetic inspection model for measuring the thickness of the nickel layer on the opposite side of the nickel-coated steel plate. Since the permeability of the mother steel (SS400 steel) is higher than that of the nickel layer, for the magnetic flux to permeate up to the nickel layer on the opposite side of the steel plate, the impression of a large magnetic field is necessary. This inspection sensor is composed of a magnetic yoke (laminated silicon steel plates), an excitation coil for the pulsed magnetic field, and a search coil. The excitation and search coils have 60 and 20 turns, respectively. The distance (lift-off) between the magnetic yoke and the surface of the steel plate is equal to 0.1 $\mathrm{mm}$. The excitation current for the pulsed magnetic field is $5 \mathrm{~A}$, and the excitation time is $1 \mathrm{~s}$, as shown in Fig. 4. The thickness of the mother steel plate (SS400 steel) is constant at $5 \mathrm{~mm}$, and the thickness of the nickel layer on the opposite side is changed from 0 to $0.2 \mathrm{~mm}$.

\subsection{D FEM analysis taking account of hysteresis loops}

The magnetic field is analyzed by 3D edge-based hexahedral nonlinear FEM and the stepby-step method considering the hysteresis magnetization curves and conductivities of both

Table 1

Conductivities of nickel and mother steel (SS400 steel).

\begin{tabular}{lcc}
\hline & Nickel & Mother steel (SS400 steel) \\
\hline Conductivity (S/m) & $12 \times 10^{6}$ & $7.5 \times 10^{6}$
\end{tabular}

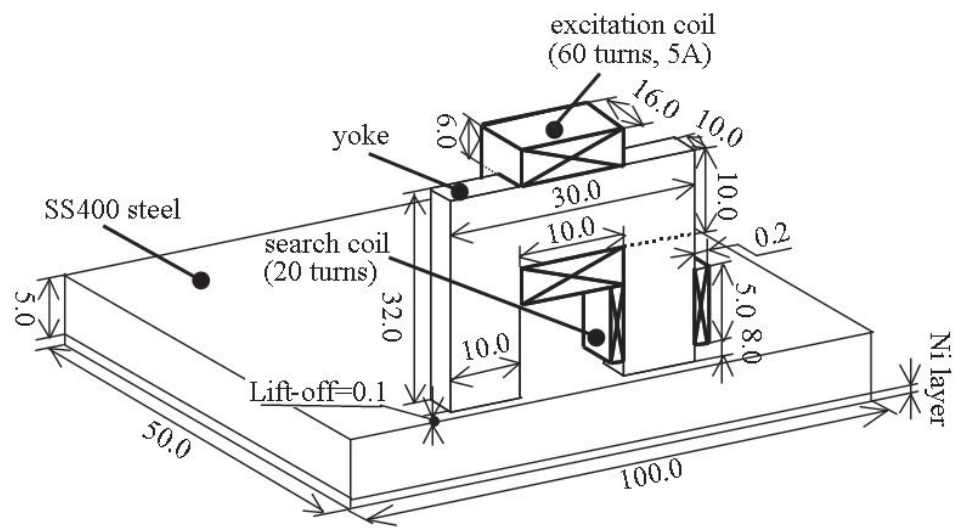

Fig. 3. Proposed electromagnetic inspection model (1/2 domain, unit: $\mathrm{mm})$.

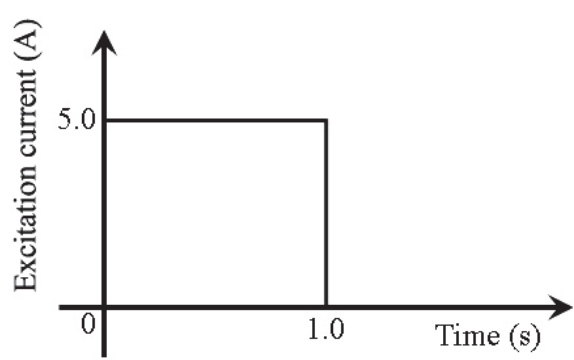

Fig. 4. Excitation current waveform. 
the mother steel (SS400 steel) and nickel layer in the nickel-coated steel plate. An initial magnetization curve of the magnetic yoke is also considered, but the eddy current in it is neglected.

The basic equation with eddy current for the $\boldsymbol{A}-\phi$ method is

$$
\begin{gathered}
\operatorname{rot}(v \operatorname{rot} \boldsymbol{A})=\boldsymbol{J}_{o}-\sigma\left(\frac{\partial \boldsymbol{A}}{\partial t}+\operatorname{grad} \phi\right) \\
\operatorname{div}\left\{-\sigma\left(\frac{\partial \boldsymbol{A}}{\partial t}+\operatorname{grad} \phi\right)\right\}=0
\end{gathered}
$$

where $\boldsymbol{A}$ is the magnetic vector potential, $\phi$ is the scalar potential, $v$ is the reluctivity, $\boldsymbol{J}_{o}$ is the current density, and $\sigma$ is the conductivity. The flux and eddy current are analyzed by taking into account the hysteresis curves and conductivities of both nickel and SS400 steel. The Newton-Raphson $(N-R)$ method is used for nonlinear iteration. The changes in $\delta \boldsymbol{A}$ and $\delta \phi$ of $\boldsymbol{A}$ and $\phi$, respectively, are obtained by solving the following equation:

$$
\left[\begin{array}{cc}
\frac{\partial G_{i}}{\partial A_{k}} & \frac{\partial G_{i}}{\partial \phi_{j}} \\
\frac{\partial \eta_{m}}{\partial A_{k}} & \frac{\partial \eta_{m}}{\partial \phi_{j}}
\end{array}\right]\left\{\begin{array}{l}
\partial A_{k} \\
\partial \phi_{j}
\end{array}\right\}=\left\{\begin{array}{l}
-G_{i} \\
-\eta_{m}
\end{array}\right\},
$$

where $G_{i}$ and $\eta_{m}$ are the residues of (1) and (2), respectively. $\partial G_{i} / \partial A_{k}$ includes the derivative term of the reluctivity, $\partial v / \partial \boldsymbol{B}^{2}$. The time interval $\Delta t$ of the step-by-step method is chosen as $1.0 \times 10^{-3} \mathrm{~s}$. The conditions of calculation and measurement are shown in Table 2 .

Figure 5 shows the distribution of flux density inside the steel plate with and without the nickel layer and the magnetic yoke near a search coil when the pulsed magnetic field of 5 A is impressed. The thickness of the nickel layer in the nickel-coated steel plate is $0.2 \mathrm{~mm}$. This figure denotes that magnetic flux permeates up to the opposite side of the steel plate. In addition, the maximum flux density of the surface layer in the steel plate slightly decreases if there is a nickel layer on the opposite side of the steel plate. This is because the impressed magnetic field from the magnetic yoke is distributed up to the nickel layer on the opposite side of the steel plate.

Figure 6 shows the distribution of flux density in the steel plate with and without the nickel layer after $0.001 \mathrm{~s}$ from the time when the pulsed magnetic field is cut. This figure denotes that, since it is immediately after the impressed magnetic field is cut, the eddy current is generated

Table 2

Conditions of calculation and measurement.

\begin{tabular}{cccc}
\hline Excitation coil & Search coil & Nodes and elements & Convergence criterion \\
\hline $\begin{array}{c}60 \text { turns, } 5 \mathrm{~A} \\
\text { (exciting time: } 1 \mathrm{~s})\end{array}$ & 20 turns & 48510 and 44268 & $\begin{array}{c}N-R \text { method: } 1.0 \times 10^{-6} \mathrm{~T} \\
\text { ICCG method: } 1.0 \times 10^{-6}\end{array}$ \\
\hline
\end{tabular}




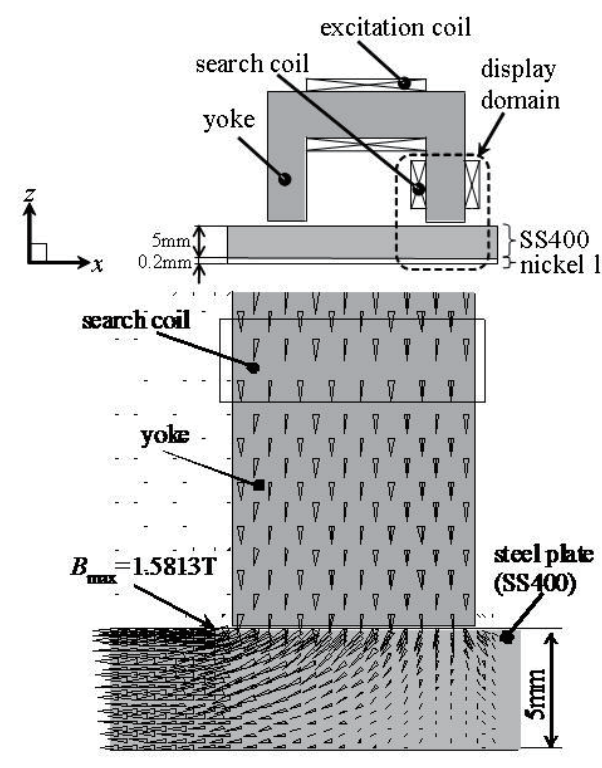

(a)

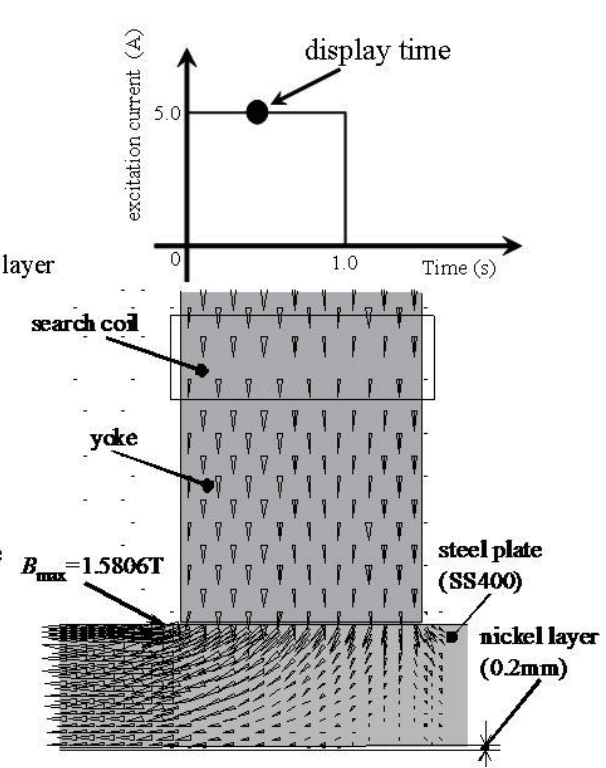

(b)

Fig. 5. Distribution of flux density inside the steel plate (a) without and (b) with the nickel layer when the pulsed magnetic field of $5 \mathrm{~A}$ is impressed.

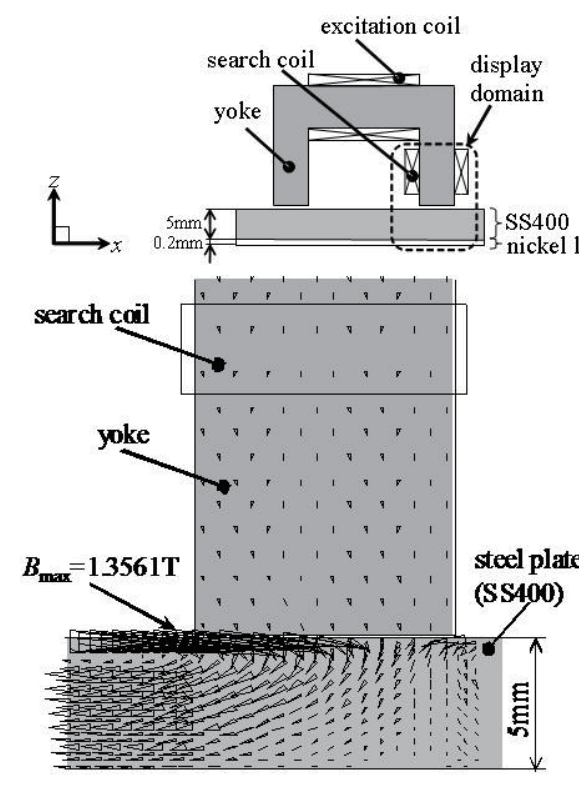

(a)
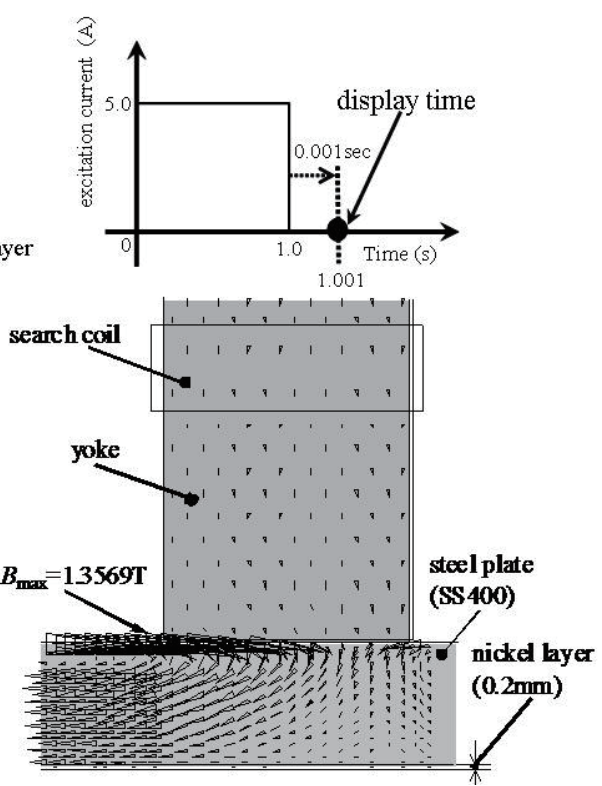

(b)

Fig. 6. Distribution of flux density inside the steel plate (a) without and (b) with the nickel layer at the time of 0.001 $\mathrm{s}$ after the pulse magnetic field is cut.

in the steel plate. Then, a large reverse-direction magnetic field is distributed on the surface of the steel plate. Moreover, the maximum flux density in the steel plate with the nickel layer is slightly higher than that in the steel plate without the nickel layer. This is because there is a ferromagnetic nickel layer with a conductivity higher than that of the mother steel (SS400 steel). 
Figure 7 shows the calculated distribution of the flux density $B_{z}$ in a search coil after 0.001 $\mathrm{s}$ from the time when the pulsed magnetic field is cut and the thickness of the nickel layer is increased. This figure denotes that the flux density $B_{z}$ in the search coil increases when the thickness of the nickel layer on the opposite side of the steel plate is increased. This is because the reverse-direction flux density in the steel plate is increased since the eddy current is increased by the increase of the domain of the ferromagnetic nickel layer with high conductivity.

\subsection{Evaluation by experiment}

In this research, the inspection method is experimentally verified. Figure 8 shows the waveform of the output voltage in a search coil after the impressed magnetic field is cut when the thickness of the nickel layer is increased. As for the vertical axis of the figure, the rate of change in output voltage after the impressed magnetic field is cut is shown. This figure denotes that the rate of change in output voltage increases near $0.0018 \mathrm{~s}$ after the impressed magnetic field is cut when the thickness of the nickel layer is increased. This figure shows that the thickness of the nickel plate is evaluated using this changing waveform of the output voltage.

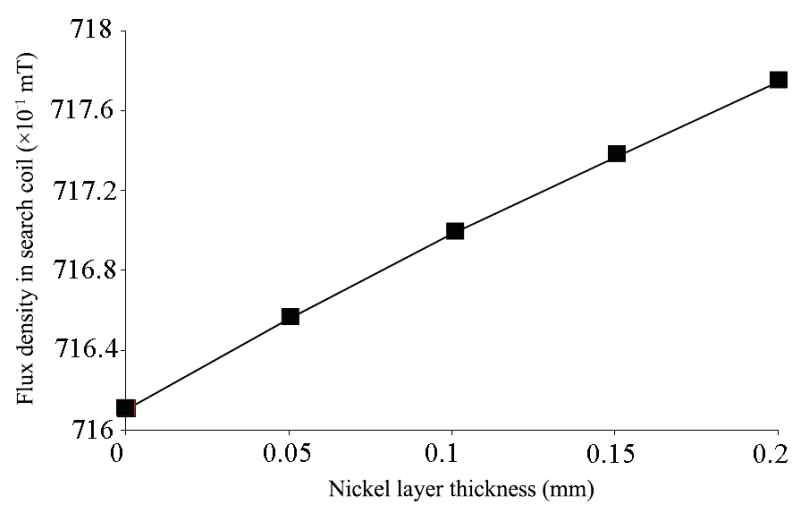

Fig. 7. Calculated distribution of flux density $B_{z}$ in a search coil $0.001 \mathrm{~s}$ after the impressed magnetic field is cut.

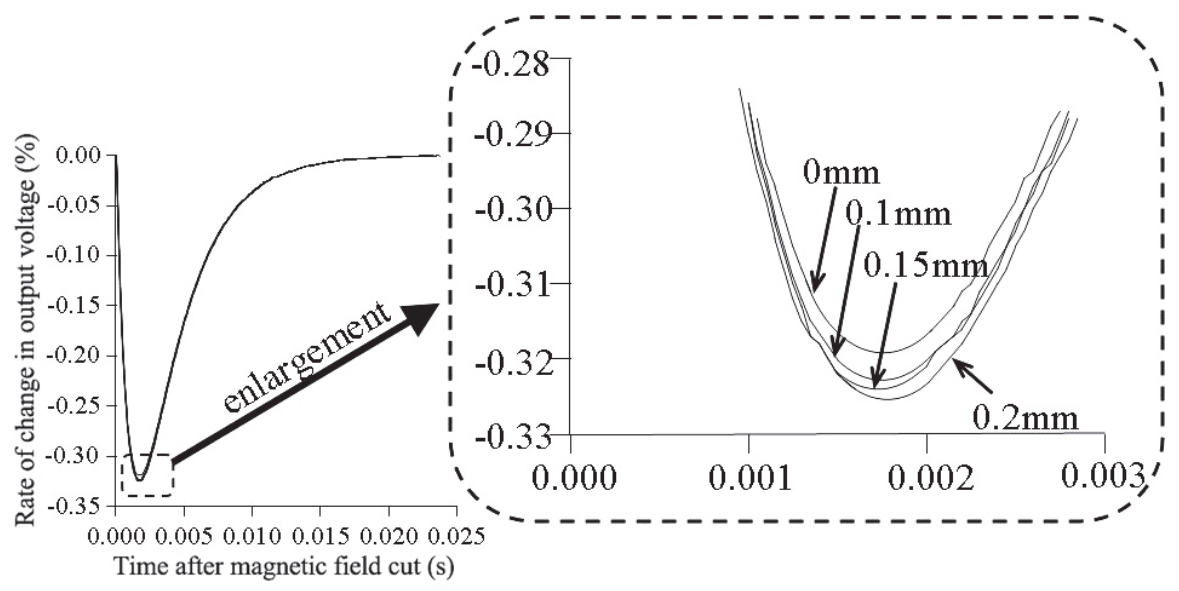

Fig. 8. Waveform of the output voltage in a search coil after the impressed magnetic field is cut at each thickness of the nickel layer on the opposite side of a steel plate (measured results). 


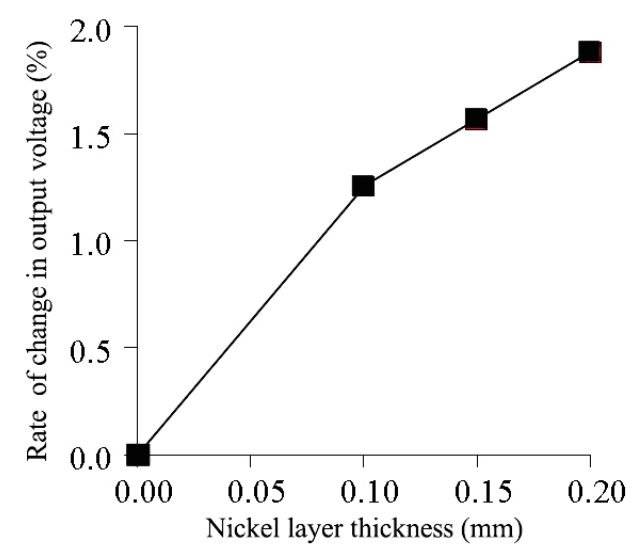

Fig. 9. Absolute value of the rate of change in output voltage in a search coil at the time of $0.0018 \mathrm{~s}$ after the impressed magnetic field is cut when the thickness of the nickel layer is increased (measured results).

Figure 9 shows the absolute value of the rate of change in output voltage in a search coil 0.0018 $\mathrm{s}$ after the impressed magnetic field is cut when the thickness of the nickel layer is increased. As for the vertical axis of the figure, the output voltage of a steel plate without the nickel layer is taken as the standard. This figure denotes that the absolute value of the output voltage increases when the thickness of the nickel layer is increased. Therefore, the thickness of the nickel plate on the opposite side of the nickel-coated steel plate can be evaluated using the output voltage in a search coil $0.0018 \mathrm{~s}$ after the impressed magnetic field is cut.

\section{Conclusions}

The results obtained are summarized as follows:

(1) The hysteresis magnetization curves and conductivities of the nickel and mother steel (SS400 steel) in the nickel-coated steel plate are measured. The permeability of nickel is lower than that of the SS400 mother steel, and the conductivity of nickel is 1.6 times that of SS400 steel.

(2) The behavior of flux density inside the nickel-coated steel plate in the thickness inspection method of the nickel layer on the opposite side using the pulsed magnetic field is calculated by 3D nonlinear FEM considering the hysteresis magnetization curve. Since the conductivity of nickel is higher than that of the SS400 mother steel, the reverse-direction magnetic field induced by the generated eddy current increases when the thickness of the nickel layer is increased. Therefore, it is possible to measure the thickness of the nickel layer on the opposite side of the steel plate using the pulsed magnetic field.

In this research, the signal change caused by the thickness of nickel is small. Moreover, the experiment and FEM analysis values are not in agreement. In addition, it is not easy to detect the thickness of the nickel layer on the opposite side and the effect of the inspection signal on the nonuniformity of permeability and remnant magnetization in an actual large rolled steel plate in a paper mill. These improvements and a more practical inspection technique are future research subjects. 


\section{References}

1 H. C. Yang and C. C. Tai: Meas. Sci. Technol. 13 (2002) 1259.

2 A. R. Shashikala, A. K. Sharma, and D. R. Bhandari: Sol. Energy Mater. Sol. Cells 91 (2007) 629.

3 V. Saxena, R. U. Rani, and A. K. Sharma: Surf. Coat. Technol. 201 (2006) 855.

4 W. E. Windes, J. Zimmerman, and I. E. Reimanis: Surf. Coat. Technol. 157 (2002) 267.

5 G. E. Staudt: AMP J. Technol. 3 (1993) 85.

6 Y. Gotoh, K. Sakurai, N. Takaoka, Y. Misaka, K. Kawasaki, and N. Takahashi: IEEE Trans. Magn. 47 (2011) 3546.

7 T. Nakata, Y. Kawase, and N. Nakano: IEEE Trans. Magn. 23 (1987) 2596.

\section{About the Authors}

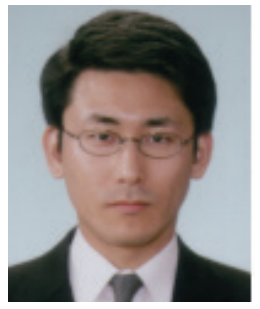

Yuji Gotoh received his B.E. and M.E. degrees from Polytechnic University, Japan, in 1996 and 1998, respectively, and his Ph.D. degree from Okayama University, Japan, in 2002. He is presently an associate professor in the Faculty of Science and Technology, Oita University. His major interest is the development of an electromagnetic nondestructive inspection method for steel materials.

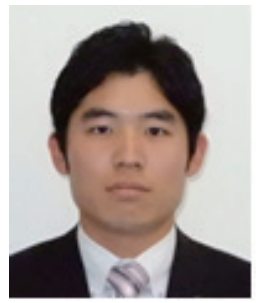

Makoto Tohara graduated from Kyushu Polytechnic College, Japan, in 2016. He received his M.E. degree from Oita University, Japan, in March, 2018. Since April, 2018, he has been conducting research as part of his doctoral course in Oita University. His research interests are nondestructive inspection using an electromagnetic phenomenon. (v18f1001@oita-u.ac.jp) 